\title{
Finite-t and target mass corrections in off-forward hard reactions
}

\author{
Vladimir Braun \\ Regensburg University, D-93040, Regensburg, Germany \\ E-mail: vladimir.braunephysik.uni-regensburg.de
}

\section{Alexander Manashov ${ }^{* i}$}

Regensburg University, D-93040, Regensburg, Germany \& Saint-Petersburg University, 199034,

St.-Petersburg, Russia

E-mail: alexander.manashov@physik.uni-regensburg.de

We describe a systematic approach $[1,2]$ to the calculation of kinematic corrections $\propto t / Q^{2}$, $\mathrm{m}^{2} / Q^{2}$ in hard exclusive processes with nonzero momentum transfer. The complete expression is derived for the time-ordered product of two electromagnetic currents that includes all kinematic corrections up to twist-four accuracy. The results are applicable to the studies of deeply-virtual Compton scattering.

Sixth International Conference on Quarks and Nuclear Physics April 16-20, 2012

Ecole Polytechnique, Palaiseau, Paris

\footnotetext{
* Speaker.

${ }^{\dagger}$ The work by A.M. was supported by the DFG, grant BR2021/5-2.
} 


\section{Introduction}

Hard exclusive scattering processes can provide one with a three-dimensional picture of the proton in longitudinal and transverse planes [3], encoded in generalized parton distributions (GPDs) $[4,6]$. One of the most important reactions in this context is Compton scattering with one real and one highly-virtual photon (DVCS). In order to probe the transverse proton structure one needs to measure the dependence of the amplitude on the target momentum transfer $t=\left(P^{\prime}-P\right)^{2}$ in a relatively broad range. Since the available photon virtualities $Q^{2}$ are limited to a few $\mathrm{GeV}^{2}$ range, corrections of the type $\propto t / Q^{2}$ (which are formally higher-twist effects), can be significant and have to be taken into account.

The theoretical description of DVCS is based on the operator product expansion (OPE) of the time-ordered product of two electromagnetic currents. In this language the GPDs appear as leading-twist operator matrix elements. The $\propto t / Q^{2}$ corrections to the amplitude are determined by the contribution of the operators of higher twist to the OPE. Among the operators of higher twist there are those which are "descendants" of the leading twist ones. They are certain total derivatives of the twist-2 operators and their matrix elements can be reduced to the matrix elements of the leading twist operators. For example, if $\mathscr{O}_{\mu_{1} \ldots \mu_{N+1}}$ is a multiplicatively renormalizable twist-two operator (symmetrized and traceless over all indices) then the operators

$$
\mathscr{O}_{1}=\partial^{2} \mathscr{O}_{\mu_{1} \ldots \mu_{N+1}}, \quad \mathscr{O}_{2}=\partial^{\mu_{1}} \mathscr{O}_{\mu_{1} \ldots \mu_{N+1}}
$$

are of twist four. The corrections to the OPE due to such operators are usually dubbed "kinematic" since they do not involve new non-perturbative parameters.

The separation of kinematic corrections $\propto t / Q^{2}$ proves, unlike target mass corrections in inclusive reactions [5], to be surprisingly difficult [6, 7, 8, 9, 10, 11, 12, 13, 14, 15]. On a technical level, the problem arises because the operator $\mathscr{O}_{2}$ has rather peculiar properties: its divergence vanishes in the free theory (the Ferrara-Grillo-Parisi-Gatto theorem [17]). It implies that with help of QCD equations of motion (EOM) $\mathscr{O}_{2}$ can be expressed in terms of quark-antiquark-gluon operators. The simplest example of such a relation is known for many years $[18,19,20]$ :

$$
\partial^{\mu} O_{\mu v}=2 \bar{q} i g G_{v \mu} \gamma^{\mu} q,
$$

where $O_{\mu v}=(1 / 2)\left[\bar{q} \gamma_{\mu} \stackrel{\leftrightarrow}{D}_{v} q+(\mu \leftrightarrow v)\right]$ is the quark part of the energy-momentum tensor. Schematically, the general structure of such relations is

$$
(\partial \mathscr{O})_{N}=\sum_{k} a_{k}^{(N)} G_{N k}
$$

where $G_{N k}$ are twist-four quark-antiquark-gluon operators and $a_{k}^{(N)}$ are the numerical coefficients. The subscript $N$ stands for the number of derivatives in $\mathscr{O}_{N}$ and the summation goes over all contributing operators. The same operators, $G_{N k}$, also appear in the OPE at the twist-four level:

$$
T\{j(x) j(0)\}^{t=4}=\sum_{N, k} c_{N, k}(x) G_{N k} .
$$

The sum in the rhs of Eq. (1.3) has to be reexpanded in terms of multiplicatively renormalized operators, $\mathscr{G}_{N, k}$, which have autonomous scale dependence

$$
\mathscr{G}_{N, k}=\sum_{k^{\prime}} \psi_{k, k^{\prime}}^{(N)} G_{N, k^{\prime}}
$$


One can obtain the coefficients $\psi_{k, k^{\prime}}^{(N)}$, at least in principle, solving the renormalization group equation (RGE) for the twist four operators, $G_{N, k}$. However, Eq. (1.2) tells us that one of the solutions of the RG equation is known without calculation. Indeed, it provides one with an explicit expression for a twist-four operator with the anomalous dimension equal to the anomalous dimension of the leading twist operator. (We ignore the contributions of $\partial^{2} \mathscr{O}_{N}$ operators in this discussion; they can be taken into account using conventional methods.)

Assuming that this solution corresponds to $k=0$ (i.e. $\mathscr{G}_{N, k=0} \equiv(\partial \mathscr{O})_{N}$ and $\psi_{k=0, k^{\prime}}^{(N)}=a_{k^{\prime}}$ ) and inverting the matrix of coefficients, $\psi_{k, k^{\prime}}^{(N)},\left(\phi^{N}=\left(\psi^{(N)}\right)^{-1}\right)$ we can write the expansion of an arbitrary twist-four operator in terms of the multiplicatively renormalizable ones

$$
G_{N, k}=\phi_{k, 0}^{(N)}(\partial \mathscr{O})_{N}+\sum_{k^{\prime} \neq 0} \phi_{k, k^{\prime}}^{(N)} \mathscr{G}_{N, k^{\prime}}
$$

Inserting (1.5) into (1.3) one separates the contribution of the operator $(\partial \mathscr{O})_{N}$ to the OPE

$$
T\{j(x) j(0)\}^{\mathrm{tw}-4}=\sum_{N, k} c_{N, k}(x) \phi_{k, 0}^{(N)}(\partial \mathscr{O})_{N}+\ldots,
$$

where the ellipses stand for the "genuine" twist-four quark gluon operators. The straightforward calculation of the coefficients $\phi_{k, 0}^{(N)}$ requires knowledge of the full matrix $\psi_{k, k^{\prime}}^{(N)}$ which is not available. Fortunately, there is a way around this problem.

We start with a remark that twist-four operators in QCD come in two big groups: the socalled quasipartonic operators [21], that only involve "plus" components of the fields, and nonquasipartonic ones which also include "minus" light-cone projections. Quasipartonic operators are not relevant for the present discussion. To one loop accuracy they have an autonomous evolution and $(\partial \mathscr{O})_{N}$ does not enter the expansion of quasipartonic operators over multiplicatively renormalizable ones, Eq. (1.5). Hence the kinematic power corrections $\sim(\partial \mathscr{O})_{N}$ originate entirely from contributions of non-quasipartonic operators.

Renormalization of twist-four non-quasipartonic operators was studied recently in [22, 23]. The main result is that the corresponding RG equations are $S L(2)$-invariant and the anomalous dimension matrix for non-quasipartonic operators is hermitian with respect to a certain scalar product. This implies that different eigenvectors are mutually orthogonal, i.e.

$$
\sum_{k} \mu_{k}^{(N)} \psi_{l, k}^{(N)} \psi_{m, k}^{(N)} \sim \delta_{l, m}
$$

where $\mu_{k}^{(N)}$ is the corresponding (nontrivial) measure. This orthogonality relation together with Eq. (1.2) results in the following expression for the coefficients $\phi_{k, 0}^{(N)}$

$$
\phi_{k, 0}^{(N)}=a_{k}^{(N)}\left\|a^{(N)}\right\|^{-2},
$$

where $\left\|a^{(N)}\right\|^{2}=\sum_{k} \mu_{k}^{(N)}\left(a_{k}^{(N)}\right)^{2}$. Inserting this expression into (1.6) one ends up with the desired separation of kinematic effects. The actual derivation is done using the two-component spinor formalism in intermediate steps and requires some specific techniques of the $S L(2)$ representation theory (see Refs. [1,2] for details). 


\section{T-product of two electromagnetic currents}

To twist-four accuracy the kinematic corrections to the OPE of two electromagnetic currents $T_{\mu \nu}=i T\left\{j_{\mu}^{e m}(x) j_{\nu}^{e m}(0)\right\}$ can be cast into the form

$$
T_{\mu v}=-\frac{1}{\pi^{2} x^{4}}\left\{x^{\alpha}\left[S_{\mu \alpha v \beta} \mathbb{V}^{\beta}+i \varepsilon_{\mu v \alpha \beta} \mathbb{A}^{\beta}\right]+x^{2}\left[\left(x_{\mu} \partial_{v}+x_{v} \partial_{\mu}\right) \mathbb{X}+\left(x_{\mu} \partial_{v}-x_{v} \partial_{\mu}\right) \mathbb{Y}\right]\right\}
$$

where $\partial_{\mu}=\partial / \partial x^{\mu}, S_{\mu \alpha v \beta}=g_{\mu \alpha} g_{v \beta}+g_{v \alpha} g_{\mu \beta}-g_{\mu \nu} g_{\alpha \beta}$ and a totally antisymmetric tensor is defined such that $\varepsilon_{0123}=1$. The expansion of functions $\mathbb{V}_{\beta}$ and $\mathbb{A}_{\beta}$ starts from twist two, wheareas $\mathbb{X}$ and $\mathbb{Y}$ are already twist-four.

In order to write the result we define nonlocal (light-ray) vector and axial-vector operators as the generating functions for local twist-two operators

$$
O\left(z_{1} x, z_{2} x\right)=\left[\bar{q}\left(z_{1} x\right) \not x\left(\gamma_{5}\right) Q^{2} q\left(z_{2} x\right)\right]_{\text {l.t. }} .
$$

Here $x_{\mu}$ is an arbitrary four-vector (not necessarily light-like), $z_{1}, z_{2}$ are real numbers, $Q$ is the matrix of quark electromagnetic charges and the Wilson line between the quark fields is implied. The leading-twist projector $[\ldots]_{l . t .}$ stands for the subtraction of traces of the local operators so that

$$
O\left(z_{1} x, z_{2} x\right)=\sum_{N} \frac{x_{\mu} x_{\mu_{1}} \ldots x_{\mu_{N}}}{N !}\left\{\bar{q}(0) \gamma_{\mu}\left[z_{1} \overleftarrow{D}_{\mu_{1}}+z_{2} \vec{D}_{\mu_{1}}\right] \ldots\left[z_{1} \overleftarrow{D}_{\mu_{N}}+z_{2} \vec{D}_{\mu_{N}}\right] Q^{2} q(0)-\text { traces }\right\}
$$

As a consequence the nonlocal operator (2.2) satisfies the Laplace equation $\partial_{x}^{2} O\left(z_{1} x, z_{2} x\right)=0$. The explicit form of the projector $[\ldots]_{\text {l.t. }}$ is irrelevant for what follows. Useful representations can be found e.g. in [11, 24].

Thanks to crossing symmetry the vector (axial-vector) operators enter the OPE (2.1) antisymmetrized (symmetrized) over the quark and antiquark positions so it is convenient to define:

$$
\begin{aligned}
& O_{V}\left(z_{1}, z_{2}\right)=\left[\bar{q}\left(z_{1} x\right) \ngtr Q^{2} q\left(z_{2} x\right)\right]_{\text {l.t. }}-\left(z_{1} \leftrightarrow z_{2}\right), \\
& O_{A}\left(z_{1}, z_{2}\right)=\left[\bar{q}\left(z_{1} x\right) \ngtr \gamma_{5} Q^{2} q\left(z_{2} x\right)\right]_{\text {l.t. }}+\left(z_{1} \leftrightarrow z_{2}\right) .
\end{aligned}
$$

The leading-twist expressions for the functions $\mathbb{V}, \mathbb{A}$ are well known and can be written as (cf. [24])

$$
\mathbb{V}_{\mu}^{t=2}=\frac{1}{2} \partial_{\mu} \int_{0}^{1} d u O_{V}(u, 0), \quad \mathbb{A}_{\mu}^{t=2}=\frac{1}{2} \partial_{\mu} \int_{0}^{1} d u O_{A}(u, 0)
$$

Note that the separation of the leading-twist terms $[\ldots]_{l . t .}$ from the nonlocal operators produces a series of kinematic power corrections to the amplitudes, which are similar to Nachtmann target mass corrections in DIS [5]. Such corrections are discussed in detail in [10, 11, 9, 12, 13, 14, 15].

For the twist-three functions we obtain (cf. $[8,9,10,16])$

$$
\mathbb{V}(\mathbb{A})_{\mu}^{t=3}=\left[i \mathbf{P}^{v}, \int_{0}^{1} d u\left\{i \varepsilon_{\mu \alpha \beta v} x^{\alpha} \partial^{\beta} \widetilde{O}_{A(V)}(u)+\left(S_{\mu \alpha v \beta} x^{\alpha} \partial^{\beta}+\ln u \partial^{\mu} x^{2} \partial^{v}\right) \widetilde{O}_{V(A)}(u)\right\}\right],
$$

Here $\mathbf{P}_{v}$ is the momentum operator, $\left[i \mathbf{P}_{v}, q(y)\right]=\partial_{v} q(y)$, and we used the notation

$$
\widetilde{O}_{V}(A)(z)=\frac{1}{4} \int_{0}^{z} d w O_{V(A)}(z, w) .
$$


One can verify that $x^{\mu} \mathbb{V}_{\mu}^{t=3}=\partial^{\mu} \mathbb{V}_{\mu}^{t=3}=0$ and similarly for $\mathbb{A}_{\mu}^{t=3}$. The term $\sim \ln u$ in Eq. (2.5) is itself twist-four and can be omitted if the calculation is done to twist-three accuracy. The resulting simplified expression is in agreement with Refs. [8, 9].

The twist-four contributions to Eq. (2.1) is our main result. To present it in a compact form we define new nonlocal operator which is the generating function for the local operators $(\partial \mathscr{O})_{N}$

$$
\mathscr{R}_{V(A)}\left(z_{1}, z_{2}\right)=\int_{z_{2}}^{z_{1}} d w \int_{z_{2}}^{w} \frac{d w^{\prime}}{z_{12}} \frac{w^{\prime}-z_{2}}{z_{1}-w^{\prime}} \times\left[\frac{1}{2} S_{+} \mathscr{O}_{1}^{V(A)}\left(w, w^{\prime}\right)-\left(S_{0}-1\right) \mathscr{O}_{2}^{V(A)}\left(w, w^{\prime}\right)\right],
$$

where $S_{+}, S_{0}$ are differential operators

$$
S_{+}=w^{2} \partial_{w}+2 w+w^{\prime 2} \partial_{w^{\prime}}+2 w^{\prime}, \quad S_{0}=w \partial_{w}+w^{\prime} \partial_{w^{\prime}}+2
$$

and the operators $\mathscr{O}_{1,2}$ are defined in terms of the leading-twist operator $\mathscr{O}_{++}^{t=2}\left(z_{1}, z_{2}\right)$ as follows:

$$
\mathscr{O}_{1}^{V(A)}\left(w, w^{\prime}\right)=\left[i \mathbf{P}^{\mu},\left[i \mathbf{P}_{\mu}, \mathscr{O}_{V(A)}^{t=2}\left(w, w^{\prime}\right)\right]\right], \quad \mathscr{O}_{2}^{V(A)}\left(w, w^{\prime}\right)=\left[i \mathbf{P}^{\mu}, \frac{\partial}{\partial x^{\mu}} \mathscr{O}_{V(A)}^{t=2}\left(w, w^{\prime}\right)\right] .
$$

In these notations the the twist four functions $\mathbb{X}$ and $\mathbb{Y}$ take the form

$$
\mathbb{X}=-\frac{1}{8} \int_{0}^{1} \frac{d u}{u^{2}}\left[\mathscr{R}_{V}(u, 0)-\mathscr{R}_{V}(0, u)\right], \quad \mathbb{Y}=\frac{1}{8} \int_{0}^{1} \frac{d u}{u^{2}}\left[\mathscr{R}_{V}(u, 0)+\mathscr{R}_{V}(0, u)\right] .
$$

Similarly, the functions $\mathbb{V}_{\mu}^{t=4}$ and $\mathbb{A}_{\mu}^{t=4}$ can be written as follows

$$
\mathbb{V}_{\mu}^{t=4}=x^{2}\left(\partial_{\mu} \mathbb{V}+x^{\mu} \Delta \mathbb{V}\right), \quad \mathbb{A}_{\mu}^{t=4}=x^{2} \partial_{\mu} \mathbb{A}
$$

where

$$
\begin{aligned}
\Delta \mathbb{V} & =-\frac{1}{4} \int_{0}^{1} d u \mathscr{R}_{V}(u, 0), \\
\mathbb{V} & =-\frac{1}{8}\left(\mathscr{R}_{V}(0,1)+\int_{0}^{1} \frac{d u}{u^{2}}\left[\mathscr{R}_{V}(0, u)-\left(1-u^{2}\right) \mathscr{R}_{V}(u, 0)\right]\right), \\
\mathbb{A} & =\frac{1}{8}\left(\mathscr{R}_{A}(0,1)+\int_{0}^{1} \frac{d u}{u^{2}}\left[\mathscr{R}_{A}(0, u)+\left(1-u^{2}\right) \mathscr{R}_{A}(u, 0)\right]\right) .
\end{aligned}
$$

The OPE of the electromagnetic currents $i T\left\{j_{\mu}^{e m}\left(z_{1} x\right) j_{v}^{e m}\left(z_{2} x\right)\right\}$ for arbitrary $z_{1}, z_{2}$ is more complicated and can be found in Ref.[2]. We note also that conservation of the electromagnetic current implies that $\partial^{\mu} T_{\mu v}(x)=0$ and $\partial^{v} T_{\mu v}(x)=i\left[\mathbf{P}^{v}, T_{\mu v}(x)\right]$. We have checked that these identities are satisfied up to twist-5 terms.

\section{Conclusions}

We have given a complete expression for the time-ordered product of two electromagnetic currents that resums all kinematic corrections related to quark GPDs to twist-four accuracy. The results can be applied to various two-photon processes, e.g. to the studies of deeply-virtual Compton scattering and $\gamma^{*} \rightarrow(\pi, \eta, \ldots)+\gamma$ transition form factors. The twist-four terms calculated in this work give rise to both a $\propto t / Q^{2}$ correction and the target mass correction $\propto m^{2} / Q^{2}$ for DVCS, whereas for the transition form factors these two effects are indistinguishable as there is only one scale. The main remaining question is whether QCD factorization itself is valid in such reactions to twist-four accuracy, at least for kinematic contributions. 


\section{References}

[1] V. M. Braun, A. N. Manashov, Phys. Rev. Lett. 107 (2011) 202001

[2] V. M. Braun and A. N. Manashov, JHEP 1201 (2012) 085 [arXiv:1111.6765 [hep-ph]].

[3] M. Burkardt, Phys. Rev. D 62 (2000) 071503

[4] M. Diehl, Phys. Rep. 388 (2003) 41

[5] O. Nachtmann, Nucl. Phys. B 63 (1973) 237

[6] A. V. Belitsky, A. V. Radyushkin, Phys. Rep. 418 (2005) 1

[7] J. Blumlein, D. Robaschik, Nucl. Phys. B 581 (2000) 449

[8] A. V. Radyushkin, C. Weiss, Phys. Rev. D 63 (2001) 114012

[9] A. V. Belitsky, D. Mueller, Nucl. Phys. B 589 (2000) 611

[10] N. Kivel, M. V. Polyakov, A. Schäfer, O. V. Teryaev, Phys. Lett. B 497 (2001) 73

[11] A. V. Belitsky, D. Mueller, Phys. Lett. B 507 (2001) 173

[12] A. V. Belitsky, D. Mueller, Phys. Rev. D 82 (2010) 074010

[13] B. Geyer, D. Robaschik, J. Eilers, Nucl. Phys. B 704 (2005) 279

[14] J. Blumlein, B. Geyer, D. Robaschik, Nucl. Phys. B 755 (2006) 112

[15] J. Blumlein, D. Robaschik, B. Geyer, Eur. Phys. J. C 61 (2009) 279

[16] I. V. Anikin, B. Pire and O. V. Teryaev, Phys. Rev. D 62 (2000) 071502

[17] S. Ferrara, A. F. Grillo, G. Parisi, R. Gatto, Phys. Lett. B 38 (1972) 333

[18] A. V. Kolesnichenko, Yad. Fiz. 39 (1984) 1527

[19] V. M. Braun, A. Lenz, Phys. Rev. D 70 (2004) 074020

[20] I. V. Anikin, B. Pire, L. Szymanowski, O. V. Teryaev, S. Wallon, Phys. Rev. D 71 (2005) 034021

[21] A. P. Bukhvostov, G. V. Frolov, L. N. Lipatov, E. A. Kuraev, Nucl. Phys. B 258 (1985) 601

[22] V. M. Braun, A. N. Manashov, J. Rohrwild, Nucl. Phys. B 807 (2009) 89

[23] V. M. Braun, A. N. Manashov, J. Rohrwild, Nucl. Phys. B 826 (2010) 235

[24] I. I. Balitsky, V. M. Braun, Nucl. Phys. B 311 (1989) 541

[25] V. M. Braun, G. P. Korchemsky, D. Müller, Prog. Part. Nucl. Phys. 51 (2003) 311 